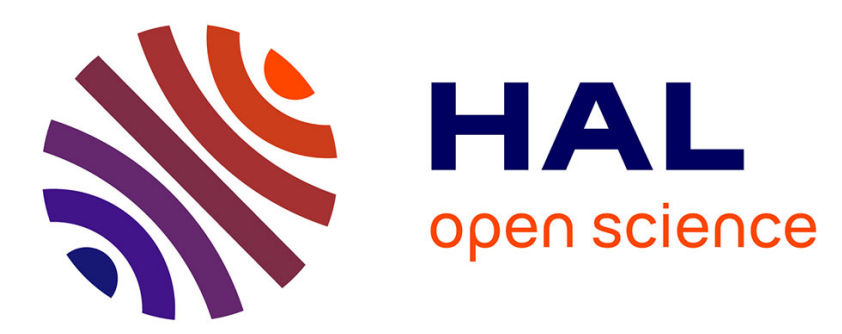

\title{
Domain of attraction estimation of cancer chemotherapy model affected by state proportional uncertainty
}

\author{
Rachid Riah, Mirko Fiacchini, Mazen Alamir
}

\section{To cite this version:}

Rachid Riah, Mirko Fiacchini, Mazen Alamir. Domain of attraction estimation of cancer chemotherapy model affected by state proportional uncertainty. ECC 2016 - 15th European Control Conference, Jun 2016, Aalborg, Denmark. hal-01298958v2

\section{HAL Id: hal-01298958 \\ https://hal.science/hal-01298958v2}

Submitted on 16 Dec 2016

HAL is a multi-disciplinary open access archive for the deposit and dissemination of scientific research documents, whether they are published or not. The documents may come from teaching and research institutions in France or abroad, or from public or private research centers.
L'archive ouverte pluridisciplinaire HAL, est destinée au dépôt et à la diffusion de documents scientifiques de niveau recherche, publiés ou non, émanant des établissements d'enseignement et de recherche français ou étrangers, des laboratoires publics ou privés. 


\title{
Domain of attraction estimation of cancer chemotherapy model affected by state proportional uncertainty
}

\author{
Rachid Riah $^{1}$, Mirko Fiacchini ${ }^{1}$ and Mazen Alamir ${ }^{1}$
}

\begin{abstract}
This paper proposes an analytical method for the characterisation of the set of all initial tumor and normal states of a cancer dynamical model for which there exists a successful drug administration therapy. The dynamical model considered simulates the behaviour of the tumor and the normal cells populations growth in presence of a chemotherapy drug. This model is assumed to be affected by a state proportional uncertainty. The successful drug administration therapy is attained by respecting the tumor eradication and avoiding the lower admissible bound of the normal cells population which is viewed as an indicator of the patients health. The problem is posed in terms of domain of attraction computation and is solved by applying set theory and invariance tools.
\end{abstract}

Keywords- Cancer, Chemotherapy, Uncertainty, Invariance, Set theory.

\section{INTRODUCTION}

On cancer research, the mathematical modeling is viewed as the pillar that have a big impact on the success of the cancer treatment. Some of the models are based on the evolution of different cells, see [1], [9], [14], [18]. Such models may incorporate the effect of external drugs on the evolution of the cancer, as chemotherapy and immunotherapy drug or their combination. In the recent years, mathematical models of cancer have been widely used also for controlbased tumoral therapy design, applying optimal control [8], [16], or feedback control, see [4], [7].

In this paper, the cancer chemotherapy model to be studied is inspired from the work [1], [18]. It represents the evolution of normal and tumor cells populations under chemotherapy drug therapy. In [1], this model is used to represent the evolution of acute myeloblastic leukemia (AML) and in [18] it has been used to simulate the cancer evolution. The normal cells represent a part of the innate immune system of the human body and can be interpreted as the aggregation of NK cells, CD8+ cells and a circulating lymphocytes (or white blood cells) as defined in [7].

The uncertainties affecting the cancer models, either parametric [3], or additive are crucial for the analysis and control design in order to find the successful drug administration therapy. Therefore, the main objective of the paper, whose preliminary version is [19], consists in the development of a new method for chemotherapy design considering that the model is affected by state proportional uncertainties. The state proportional uncertainties are the uncertainties which affect proportionally the dynamic of each state of the model.

\footnotetext{
This work was supported by the French national project CATS-INSERM.

1 GIPSA-lab, Grenoble Campus, 11 rue des Mathématiques, BP 46, 38402 Saint Martin d'Hères Cedex, France. \{rachid.riah, mirko.fiacchini, mazen.alamir\}@gipsa-lab.fr
}

This method is based on the properties of robust invariant sets for Convex Difference Inclusions (CDI) systems.

In this paper, our contribution is substantially based on set theory and invariance for control. Stability and robustness will be addressed using the properties of invariant sets. Many results regarding invariance and related topics have been provided in the literature, see [5], [13], [15], [11] and [6]. Invariance is related to reachability [17], and viability. Typically, the viability kernel is much harder to compute [21].

The new method provides the domain of attraction of the cancer chemotherapy model affected by a state proportional uncertainty. This domain contains all tumor and normal states for which a set of admissible administration chemotherapy profile exists, that drives theses states to the safe region. The safe region is defined to be the set for which the number of tumor cells is small enough and the normal cells number higher than the minimal admitted level. A minimal level is imposed as a health condition.

The paper is organized as follows: First the mathematical model of cancer is recalled in Section II. Section III presents the problem statement addressed in the paper. The properties and numerical methods related to controlled robust invariant sets and convex difference inclusions systems are given in Section IV. The application to the cancer problem of the methods developed in Section IV is proposed in Section V. Section VI illustrates the results given in Section V. Section VII ends the paper providing conclusions and giving hints for future investigations.

Notation: Given $n \in \mathbb{N}$, define $\mathbb{N}_{n}=\{m \in \mathbb{N}: 1 \leq m \leq n\}$. Given $A \in \mathbb{R}^{n \times m}, A_{i}$ with $i \in \mathbb{N}_{n}$ denotes its $i$-th row, $A_{i, j}$ with $i \in \mathbb{N}_{n}$ and $j \in \mathbb{N}_{m}$ a value that corresponds to the $i$-th row and the $j$-th column.

\section{MATHEMATICAL CANCER MODEL}

We consider the mathematical model that has been developed in [18], [1] and [2] in order to describe the dynamical behaviour of the normal and tumor cells populations under a chemotherapy drug. This model involves the following cells populations

- L: tumor cells population,

- $\mathrm{N}$ : normal cells population.

The assumptions considered on the model are given in the following, see [18], [1] and [2] for more details.

1) The growth dynamics of both tumor and normal cells populations are the same anywhere in the body;

2) The tumor and normal cells populations follow a process of Gompertizian growth; 
3) The tumor cells exhibit a negative and inhibiting effect on the growth and development of the normal cells;

4) The chemotherapy drug kills both tumor and normal cells and affects the normal more than the tumor cells;

5) The drug spreads instantaneously within the body.

The resulting model, based on the above assumptions, is defined as follows

$$
\begin{array}{ll}
\dot{L}=\alpha L \ln \frac{\theta_{L}}{L}-\Pi_{1}(u) L, & L(0)=L_{0}, \\
\dot{N}=\beta N \ln \frac{\theta_{N}}{N}-\Xi(L) N-\Pi_{2}(u) N, & N(0)=N_{0},
\end{array}
$$

where the description of each term is given in Table I.

\begin{tabular}{ll}
\hline Term & Description \\
\hline$\alpha$ & Tumor growth rate \\
$\theta_{L}$ & Greatest size of the tumor cells population \\
$\alpha L \ln \frac{\theta_{L}}{L}$ & Gompertizian tumor growth \\
$\Pi_{1}(u) L$ & Death of tumor cells due to chemotherapy \\
$\beta$ & Normal cells growth rate \\
$\theta_{N}$ & Greatest size of the normal cells population \\
$\beta N \ln \frac{\theta_{N}}{N}$ & Gompertizian normal growth \\
$\Xi(L) N$ & Stimulation of tumor cells on normal cells \\
$\Pi_{2}(u) N$ & Death of normal cells due to chemotherapy \\
\hline
\end{tabular}

TABLE I: Model terms description (1).

Following the reasonings in [1], [18] and [2], the assumptions below can be done to simplify the model.

- The function $\Xi(L)$ is taken to be linear as in [1] (i.e. $\Xi(L)=\gamma L$, where $\gamma$ is a given parameter);

- We consider that the loss function $\Pi_{1}(u)$ is linear, taking into account the saturation phenomena, i.e, $\Pi_{1}(u)=k u$, $k$ is the fraction of tumor cells that are killed due to the chemotherapy drugs;

- We assume that the loss function $\Pi_{2}(u)$ is linear, i.e, $\Pi_{1}(u)=l u, l$ is the fraction of the normal cells that are destroyed by the effects of the chemotherapy drugs (it is assumed that $l<k$ );

- We assume also that the dynamics of the cells populations are affected by state proportional uncertainties.

Physically, the state proportional uncertainties can be viewed as a disturbance that grows proportionally with the number of the cells populations. It is in fact reasonable to suppose that these uncertainties should depend on the number of the cells populations, since its effect on the dynamic of the model must be suitable for small and high number of the cells populations.

The resulting model becomes

$$
\begin{array}{ll}
\dot{L}=\alpha L \ln \frac{\theta_{L}}{L}-k u L+w_{1} L, & L(0)=L_{0}, \\
\dot{N}=\beta N \ln \frac{\theta_{N}}{N}-\gamma L N-l u N+w_{2} N, & N(0)=N_{0},
\end{array}
$$

where $\left(w_{1} w_{2}\right)^{T} \in W \subseteq \mathbb{R}^{2}$ is an unknown uncertainty belonging to a bounded set $W$. The bounded set $W$ can be taken as a box, a polyhedral set, an ellipsoidal set or their intersection.

As proposed in [18], we introduce the change of variables $x_{1}=\ln \frac{\theta_{L}}{L}$ and $x_{2}=\ln \frac{\theta_{N}}{N}$, and we get the equivalent system

$$
\begin{array}{ll}
\dot{x_{1}}=-\alpha x_{1}+k u-w_{1}, & x_{1}(0)=x_{10}, \\
\dot{x_{2}}=-\beta x_{2}+\gamma \theta_{L} e^{-x_{1}}+l u-w_{2}, & x_{2}(0)=x_{20} .
\end{array}
$$

The discrete-time system modelling the cancer evolution is obtained by sampling the continuous system (3), with numerical integration schemes using Euler's method and sampling time of $T_{s}=1$ day. It results in

$$
\begin{array}{rlrl}
x_{1}^{+} & =\left(1-T_{s} \alpha\right) x_{1}+T_{s} k u-T_{s} w_{1} & \\
& =f_{1}(x, u)-T_{s} w_{1}, & & x_{1}(0)=x_{10}, \\
x_{2}^{+} & =\left(1-T_{s} \beta\right) x_{2}+T_{s} \gamma \theta_{L} e^{-x_{1}}+T_{s} l u-T_{s} w_{2} & & \\
& =f_{2}(x, u)-T_{s} w_{2}, & & x_{2}(0)=x_{20} .
\end{array}
$$

The model (4) involves two state variables $x_{1}, x_{2}$, one manipulated variable $u$ and two uncertainties variables $w_{1}$ and $w_{2}$. Notice that in this space, the uncertainty appears as additive terms. The consistent values for the parameters involved in this model are inferred from [1] and [18], then modified to reproduce the cells evolutions given in [1] and summarized in Table II.

\section{Problem Statement}

Consider the nonlinear model (2) that describes the dynamics of the cells populations in presence of chemotherapy drug and state proportional uncertainties.

The system is subject to the following constraints on system states, control inputs and uncertainty variables.

$$
\begin{aligned}
& (L N)^{T} \in Y \subseteq \mathbb{R}^{2}, \\
& u \in U \subseteq \mathbb{R}=\left\{u \in \mathbb{R}^{m}: u_{\text {min }} \leq u \leq u_{\text {max }}\right\}, \\
& w \in W \subseteq \mathbb{R}^{2} .
\end{aligned}
$$

In cancer therapy drug administration, the main objective is to reduce the tumor cells population $L$, while maintaining the number of the normal cells population $N$ above a prescribed level, see [7], [9]. The initial values of $(L N)^{T}$ belong to $Y$. The number of the normal cells population $N$ is used in [18] as a measure of the patient health.

In this paper we would like to determine all the initial tumor and normal states for which there exists an appropriate drug injection profile $u$ satisfying the constraint (6) for all uncertainties realizations $w$ belonging to $W$. This therapy must lead to a substantial regression of the tumor size, i.e. $L$ becomes small enough, while avoiding that the health measure reaches dangerous values for the patient, i.e. $N \geq$ $N_{\min }$, with $N_{\min }$ denoting the minimal admitted value of the normal cells.

In the space of $x_{1}$ and $x_{2}$, the state constraint (5) becomes

$$
\left(\begin{array}{ll}
x_{1} & x_{2}
\end{array}\right)^{T} \in X \subseteq \mathbb{R}^{n},
$$

where $X$ is determined from the set $Y$ applying the change of variable defined above.

In this space, we would like to determine the set of the initial points $\left(x_{1}, x_{2}\right) \in X$, such that the dynamics of the system (4) can be driven to the safe region for all

\begin{tabular}{llll}
\hline param. & value & param. & value \\
\hline$\alpha$ & $3.96 \times 10^{-4}$ day $^{-1}$ & $\beta$ & $3.33 \times 10^{-2}$ day $^{-1}$ \\
$\theta_{N}$ & $1.4 \times 10^{8}$ cells $^{8}$ & $\theta_{L}$ & $3 \times 10^{8}$ cells \\
$k$ & $8 \times 10^{-2}$ & $l$ & $15 \times 10^{-3}$ \\
$\gamma$ & $10^{-9}(\text { cells.day })^{-1}$ & & \\
\hline
\end{tabular}

TABLE II: Parameters of the dynamic model (2). 
uncertainties realizations $w$ belonging to $W$, by applying an admissible chemotherapy drug profile $u$ satisfying the constraint (6). The safe region is the set in the state space $x_{1}, x_{2}$ defined by $x_{1}$ high enough and $x_{2} \leq x_{2 \text { max }}$, where $x_{2 \max }=\ln \frac{\theta_{N}}{N_{\min }}$ denotes the maximal admitted value of $x_{2}$.

These initial tumor and normal states represent the domain of attraction of the cancer system (4). Its computation is substantially based on the properties of positively invariant sets and convex inclusions, that will be introduced in the next section.

\section{CONVEX DIFFERENCE INCLUSIONS SYSTEMS AND DOMAIN OF ATTRACTION}

In this section, the method that provides the domain of attraction of a nonlinear systems is stated. This method is based on the approximation of the nonlinear system with a Convex Difference Inclusions (CDI) system and also on the properties of positively invariant sets. In practice, this method computes the maximal contractive invariant convex set as an estimation of the domain of attraction of a nonlinear system.

The convex difference inclusions systems are defined by a particular class of set valued maps as a dynamic function. The set valued maps that can be used to define this class of systems is such that, given a point in the state space, its image through the map is a convex and compact set.

Let us consider the uncertain difference inclusions system

$$
x^{+} \in \mathscr{F}_{W}(x, u)=\mathscr{F}(x, u)+W
$$

where $x \in X \subseteq \mathbb{R}^{n}$ is the state, $x^{+}$is the successor, $u \in$ $U \subseteq \mathbb{R}^{m}$ is the control input and $\mathscr{F}(\cdot, \cdot)$ is a set valued map on $\mathbb{R}^{n}$, that is $\mathscr{F}(x, u)$ represents a function which relates a set to every point $(x u)^{T} \in \mathbb{R}^{n} \times \mathbb{R}^{m}$, i.e. $\mathscr{F}(x, u) \subseteq \mathbb{R}^{n}$ for all $x \in \mathbb{R}^{n}$. The sets $X, W$ and $U$ represent the set of state constraints, the set of additive uncertainties and the set of admissible control inputs respectively. The set $W$ is an additive uncertainty bounding set satisfying the following assumption.

Assumption 1: The set $W \subset \mathbb{R}^{n}$ is compact, convex and $0 \in W$.

The interest of an uncertain difference inclusions systems lies in the fact that they can be used to approximate the uncertain nonlinear systems $x^{+}=f(x, u)+w$, i.e. $f(x, u)+$ $w \in \mathscr{F}_{W}(x, u)$ for all $x \in X$, all $w \in W$ and for all $u \in U$.

Since a robust invariant set for the CDI system is robust invariant also for the nonlinear system then the approximation may be useful in order to determine the domain of attraction of the nonlinear system. Although some conservatism is in general introduced, it is arbitrarily small for the case under analysis.

Notice that the important tool to deal with convex closed sets and convexity is the support function. For some properties of support functions readers can see [20].

Definition 1: Given a set $\Omega \subseteq \mathbb{R}^{n}$, the support function of $\Omega$ evaluated at $\eta \in \mathbb{R}^{n}$ is $\phi_{\Omega}(\eta)=\sup _{x \in \Omega} \eta^{T} x$.

The support function permits to transform the set-inclusion condition in terms of linear inequalities, see [20] for instance. From the definition of the support function, we get this property.
Property 1: Given two closed, convex sets $\Omega \subseteq \mathbb{R}^{n}$ and $\Gamma \subseteq \mathbb{R}^{n}$, then $x \in \Omega$ if and only if $\eta^{T} x \leq \phi_{\Omega}(\eta)$ for all $\eta \in \mathbb{R}^{n}$, and $\Gamma \subseteq \Omega$ if and only if $\phi_{\Gamma}(\eta) \leq \phi_{\Omega}(\eta)$, for all $\eta \in \mathbb{R}^{n}$.

In order to define the uncertain difference inclusions systems, let us introduce this assumption.

Assumption 2: Assume that the set valued map $\mathscr{F}$ determining the system dynamics (9) is such that $\mathscr{F}(x, u)$ is compact and convex for all $(x u) \in \mathbb{R}^{n} \times \mathbb{R}^{m}$ and for every $\eta \in \mathbb{R}^{n}$, the function $F(x, u, \eta): \mathbb{R}^{n} \times \mathbb{R}^{m} \times \mathbb{R}^{n} \longrightarrow \mathbb{R}$ defined as

$$
F(x, u, \eta)=\phi_{\mathscr{F}(x, u)}(\eta)=\sup _{z \in \mathscr{F}(x, u)} \eta^{T} z
$$

is convex with respect to $(x u)$ on $X \times U$.

Thus, in practice, $F(x, u, \eta)$ is the support function of the set $\mathscr{F}(x, u)$ evaluated at $\eta \in \mathbb{R}^{n}$ and then

$$
\mathscr{F}(x, u)=\left\{z \in \mathbb{R}^{n}: \quad \eta^{T} z \leq F(x, u, \eta), \quad \forall \eta \in \mathbb{R}^{n}\right\},
$$

and it is convex in $\left(\begin{array}{ll}x & u\end{array}\right)^{T}$.

Since the set-valued map $\mathscr{F}_{W}(x, u)$ determining the system (9) is the sum of two convex sets, then it is convex also with respect to $(x u)^{T} \in \mathbb{R}^{n} \times \mathbb{R}^{m}$. Thus, the set valued map determining the system (9) is such that

$$
\mathscr{F}_{W}(x, u)=\left\{z \in \mathbb{R}^{n}: \quad \eta^{T} z \leq F(x, u, \eta)+\phi_{W}(\eta), \quad \forall \eta \in \mathbb{R}^{n}\right\} .
$$

The dynamical systems (9) for which Assumption 2 holds are tightly related to the uncertain CDI systems defined in [10], [12].

Now, we recall the standard definitions of the controlled robust invariant set for the generic nonlinear system, adapted here for the case of potentially unbounded sets that do not necessarily contain the origin and set valued maps.

Definition 2 ([6]): The closed convex set $\Omega \subseteq \mathbb{R}^{n}$ is a controlled robust invariant set for the uncertain nonlinear system $x^{+}=f_{W}(x, u)=f(x, u)+w$ if for every $x \in \Omega$, there exists $u \in U$ such that $f_{W}(x, u) \in \Omega$ for all $w \in W$; it is controlled robust invariant for the CDI system (9) if for every $x \in \Omega$, there exists $u \in U$ such that $\mathscr{F}_{W}(x, u) \subseteq \Omega$.

Whatever the additive uncertainties $w \in W$ affecting the trajectory started in a controlled robust invariant set $\Omega$, there exists an admissible control input, such that this trajectory remains inside $\Omega$.

For the system (9), the robust controlled invariance of $\Omega \subseteq X$ can be tested using the controlled robust one-step backward operator, defined below.

Definition 3: Consider the closed convex set $\Omega$ and assume that Assumption 2 holds for the set valued map $\mathscr{F}(\cdot, \cdot)$ determining the dynamic system (9). The controlled robust one-step operator is defined as follows

$$
Q_{W}(\Omega, U, X)=\left\{x \in X: \quad \exists u \in U: \quad \mathscr{F}_{W}(x, u) \subseteq \Omega\right\} .
$$

Hence, the controlled robust one-step operator associates to every set $\Omega$ the set of points that are mapped inside $\Omega$ through $\mathscr{F}_{W}(\cdot, \cdot)$ applying an appropriate control input $u \in U$. Thus, by definition, a closed convex set $\Omega \subseteq X$ is robust invariant if and only if $\Omega \subseteq Q_{W}(\Omega, U, X)$ and the controlled robust one-step operator can be used to check controlled robust invariance and to compute increasing sequences of nested controlled robust invariant sets as for linear or CDI systems. Indeed, Algorithm 1, standard for generating a sequence of convex sets that converges to the domain of attraction, see [6], can be applied also in this context. 


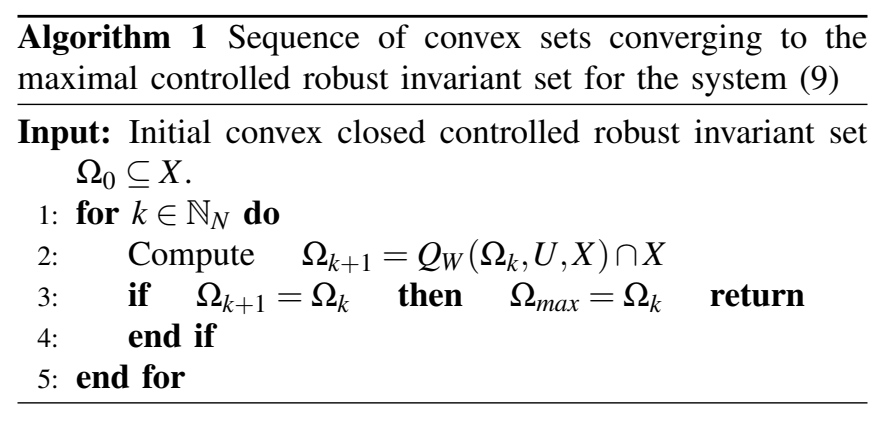

Notice that Algorithm 1 can be used starting from the set of state constraints $\Omega_{0}=X$ or from an initial controlled robust invariant set $\Omega_{0}$. Thus, in the latter case, $\Omega_{k}$ are controlled robust invariant set and converges to the domain of attraction for the system (9), and hence for all nonlinear systems approximated by (9). Finally, being our objective to have a sequence of $\Omega_{k}$ that are polyhedral, we introduce the following results, proved in [10], which is functional for this purpose.

Proposition 1: Consider the set-valued map $\mathscr{F}_{W}(x, u)$ determining the system dynamics (9). Given the state constraint set $X$, the control input set $U$ and the polytope $\Omega=\{x \in$ $\left.\mathbb{R}^{n}: H x \leq h\right\}$, with $H \in \mathbb{R}^{n_{h} \times n}$, the controlled robust onestep operator is

$$
\begin{gathered}
Q_{W}(\Omega, U, X)=\left\{x \in X: \quad \exists u \in U: \quad F\left(x, u, H_{i}^{T}\right) \leq h_{i}\right. \\
\left.-\phi_{W}\left(H_{i}^{T}\right), \quad \forall i \in \mathbb{N}_{n_{h}}\right\} .
\end{gathered}
$$

Proof: From Definition 2, we have that $x \in Q_{W}(\Omega, U, X)$ if and only if $\exists u \in U$ such that $\mathscr{F}_{W}(x, u) \subseteq \Omega$, which is the condition given to determine the controlled robust one-step operator. Thus, using the Property 1 and Eq.(12) the latter condition is equivalent to $x \in Q_{W}(\Omega, U, X)$ if and only if $\exists u \in$ $U$ such that $\phi_{\mathscr{F}}(x, u)(\eta)+\phi_{W}(\eta) \leq \phi_{\Omega}(\eta)$ for all $\eta \in \mathbb{R}^{n}$. For polyhedral case, this condition is equivalent also to $x \in$ $Q_{W}(\Omega, U, X)$ if and only if $\exists u \in U$ such that $F\left(x, u, H_{i}^{T}\right) \leq$ $h_{i}-\phi_{W}\left(H_{i}^{T}\right), \forall i \in \mathbb{N}_{n_{h}}$ which is proved in [10]. Finally, the controlled robust one-step operator can be defined as given by Eq.(14).

Notice that, as proved in [10] for general convex closed sets, for every polytopic $\Omega$, the set $Q_{W}(\Omega, U, X)$ is closed and convex. Moreover, if $F(\cdot, \cdot, \eta)$ are piecewise affine functions of $(x \quad u)^{T}$, then $Q_{W}(\Omega, U, X)$ is a polyhedron, that is the intersection of a finite number of halfspaces, for every polytopic (or polyhedral) $\Omega$.

Finally, once the controlled robust one-step operator is given, Algorithm 1 can be used to compute the domain of attraction of the nonlinear systems $x^{+}=f(x, u)+w$, with $w \in W$.

\section{CONTROL ROBUST ONE-STEP OPERATOR FOR CANCER CHEMOTHERAPY}

In this section, the controlled robust one-step operator for the uncertain cancer chemotherapy model (4) is stated. Notice that the model (4) is in the form $x^{+}=f(x, u)+w$. Then given the set $\Omega=\left\{x \in \mathbb{R}^{2}: H x \leq h\right\}$, with $H \in \mathbb{R}^{n_{h} \times 2}$, the controlled robust one-step operator of this model can be computed using Eq.(14). Eq.(14) depends on the bounding functions $F\left(x, u, H_{i}\right)$ for all $H_{i}^{T} \in \mathbb{R}^{2}, i \in \mathbb{N}_{n_{h}}$, that must first be determined.

First, we will compute the bounding functions $F(x, u, \eta)$ for all $\eta \in \mathbb{R}^{2}$. This function has to be convex with respect to $(x u)^{T}$, and such that the associated set-valued maps $\mathscr{F}(x, u)$ as $(11)$, satisfies $f(x, u) \in \mathscr{F}(x, u)$, with $f(x, u)$ in (4), for every $(x u)^{T} \in X \times U$. Thus, according to Property 1 , the functions $F(x, u, \eta)$ must satisfy the following inequality $\eta^{T} f(x, u) \leq F(x, u, \eta)$, for every $\eta \in \mathbb{R}^{2}$. Moreover, since the nonlinearity in (4) involves only $f_{2}(x, u)$ then the bounding functions of $\eta^{T} f(x, u)$ are related only to $\eta_{2}$, since the dynamics of $x_{1}$ is linear. A possible choice is

- if $\eta_{2} \geq 0: \quad F(x, u, \eta)=\eta^{T} f(x, u)$, since $F(x, u, \eta)$ is already convex in this case;

- if $\eta_{2}<0$ :

$$
\begin{aligned}
F(x, u, \eta)=\eta_{1} & \left(\left(1-T_{s} \alpha\right) x_{1}+T_{s} k u\right)+\eta_{2}\left(\left(1-T_{s} \beta\right) x_{2}\right. \\
& \left.+T_{s} \gamma \theta_{L}\left(a x_{1}+b\right)+T_{s} l u\right)
\end{aligned}
$$

with $a$ and $b$ such that $\left(a x_{1}+b\right) \leq e^{-x_{1}}$, obtained for instance as the tangent to the graph of $e^{-x_{1}}$ at one point; for all $\eta \in \mathbb{R}^{2}$.

We are interested in convex piecewise affine bounding functions for computational purposes. For this it is sufficient to replace $e^{-x_{1}}$ with a convex piecewise affine upper bound, which is easily obtainable, for the case of $\eta_{2} \geq 0$. In fact, it is sufficient to choose a set of $q \in \mathbb{N}$ parameters $c_{i} \in \mathbb{R}$, $d_{i} \in \mathbb{R}$, with $i \in \mathbb{N}_{q}$, such that, $e^{-x_{1}} \leq \max _{i \in \mathbb{N}_{q}}\left\{c_{i} x_{1}+d_{i}\right\}$, for all $x_{1} \in \mathbb{R}$. To obtain those parameters, it is sufficient to define $z_{i}$ with $i \in \mathbb{N}_{q+1}$ such that $z_{i}<z_{i+1}$ and then

$$
c_{i}=\frac{e^{-z_{i+1}}-e^{-z_{i}}}{z_{i+1}-z_{i}}, \quad d_{i}=\frac{z_{i+1} e^{-z_{i}}-z_{i} e^{-z_{i+1}}}{z_{i+1}-z_{i}}
$$

are the parameters of an affine function such that $e^{-x_{1}} \leq$ $c_{i} x_{1}+d_{i}$ for every $x_{1} \in\left[z_{i}, z_{i+1}\right]$ and $e^{-z_{i}}=c_{i} z_{i}+d_{i}$ for all $i \in \mathbb{N}_{q}$, see Figure 1 .

Remark 1: Notice that, in order to increase the precision, the number of parameters $q$ can be taken as big as desired. Hence, arbitrary precision can be attained.

Concerning the case of $\eta_{2}<0$, no modification is required with respect to (15), since the function (15) is already piecewise affine function. Then, we obtain

$$
F(x, u, \eta)=\left\{\begin{array}{cc}
\eta_{1}\left(\left(1-T_{s} \alpha\right) x_{1}+T_{s} k u\right)+\eta_{2}\left(\left(1-T_{s} \beta\right) x_{2}\right. \\
\left.+T_{s} \gamma \theta_{L} \max _{i \in \mathbb{N}_{q}}\left\{c_{i} x_{1}+d_{i}\right\}+T_{s} l u\right) & \text { if } \eta_{2} \geq 0, \\
F(x, u, \eta) \text { as in (15) } & \text { if } \eta_{2}<0 .
\end{array}\right.
$$

Figure 1 shows the convex upper bound and the concave lower bound of $e^{-x_{1}}$ implicitly used to determine $F(x, u, \eta)$. Notice that $F(x, u, \eta)$ is a convex upper bound of $\eta^{T} f(x, u)$, for all $(x u)^{T} \in X \times U$ and for all $\eta \in \mathbb{R}^{2}$. Once a convex upper bound of $\eta^{T} f(x, u)$ is determined, the controlled robust one-step operator will be given using the Proposition 1 .

Remark 2: By applying Algorithm 1, we found that the sequence of polytopes $\Omega_{k}=\left\{x \in \mathbb{R}^{n}: H^{k} x \leq h^{k}\right\}$ generated by the controlled robust one step operator never have a facet determined by $H^{k}{ }_{i}$ with $H^{k}{ }_{i, 2}<0$ except the case related to the trivial constraints $x_{2} \leq 0$. Since the latter 


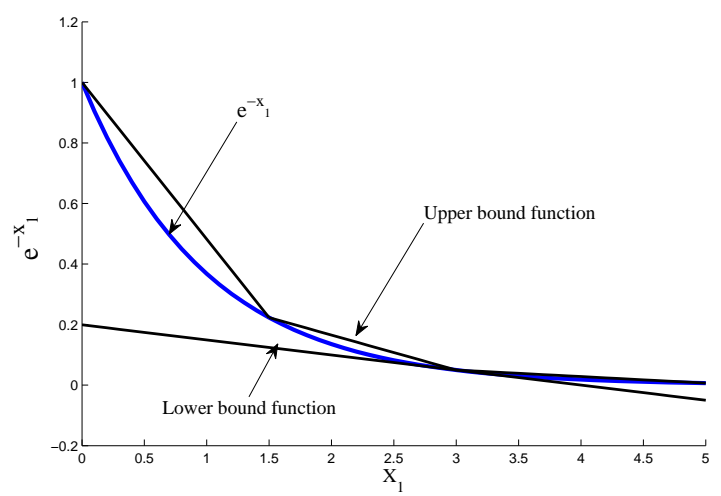

Fig. 1: Bounds of the exponential function.

constraints can be neglected in the computation, then the lower bound of $e^{-x_{1}}$ is never used. This would mean that the sequence of polytopes obtained and the domain of attraction are affected only by the mismatches between $e^{-x_{1}}$ and the piecewise function $\max _{i \in \mathbb{N}_{q}}\left\{c_{i} x_{1}+d_{i}\right\}$, mismatch that can be done arbitrarily small, as notice in Remark 1 . Hence, the desired precision can be achieved by employing sufficiently close piecewise approximations of $e^{-x_{1}}$. Consequently, the conservatism introduced by approximating the uncertain nonlinear cancer system (4) by the uncertain CDI system (9) can be reduced increasing the precision as indicated in Remark 1.

According to Remark 2, the piecewise affine bound function of $\eta^{T} f(x, u)$ to be employed is the one given for $\eta_{2} \geqslant 0$. Therefore, the related controlled robust one-step operator defined in Proposition 1becomes

$$
\begin{gathered}
Q_{W}(\Omega, U, X)=\left\{x \in X \mid \exists u \in U: \quad \forall i \in \mathbb{N}_{h_{h}}, \quad \forall j \in \mathbb{N}_{q},\right. \\
\quad\left(H_{i, 1}\left(1-T_{s} \alpha\right)+H_{i, 2} T_{s} \delta c_{j}\right) x_{1}+H_{i, 2}\left(1-T_{s} \beta\right) x_{2} \\
\left.\leq h_{i}-H_{i, 2} T_{s} \delta d_{j}-\left(H_{i, 1} T_{s} k+H_{i, 2} T_{s} l\right) u-\phi_{W}\left(H_{i}^{T}\right)\right\} .
\end{gathered}
$$

Then $Q_{W}(\Omega, U, X)$ maps polytopes in polytopes and the Algorithm 1 generates a sequence of polytopes that converge to the maximal controlled robust invariant polytopic set for the system (4).

\section{Simulations}

Let us assume that the chemotherapy drug profile is constrained to take value from $U$ such that $u_{\min }=0$ and $u_{\max }=1$. We also consider that the minimal admitted level of the normal cells is $N_{\min }=10^{7} \mathrm{Cells}$, which implies, using the change of variable that $x_{2 \max }=2.30$. Since the exact values of the model parameters are not available in the literature, the value of $N_{\min }$ is also taken approximately as the others parameters described and noted in Section II.

Let us also assume that the polyhedral set of the state constraints in the domain of $L$ and $N$ is $Y=\left\{y=(L N)^{T} \in\right.$ $\left.\mathbb{R}^{2}: \quad L \geq 0 ; N_{\min } \leq N\right\}$, which implies, using the change of variables that $X=\left\{x \in \mathbb{R}^{2}: \quad x_{1} \geq 0 ; 0 \leq x_{2} \leq x_{2 \max }\right\}$.

The state proportional uncertainty $w$ is assumed to belong to the set $W=\left\{w \in \mathbb{R}^{2}: \quad\|w\|_{2} \leqslant 0.06 ; 0 \leqslant w_{1} ; w_{2} \leqslant 0\right\}$. This set corresponds to the worst case that can affect the dynamics of the cells populations. In the model (2), the tumor cells population $L$ without chemotherapy drug diverges. The worst case for the uncertainties increases the divergence of the tumor cells population $L$ to infinity, i.e. $w_{1}$ is taken positive. The normal cells population needs to grow and the worst case of the uncertainties prevent this growth, i.e. $w_{2}$ is taken negative.

Once the set of constraint states $X$ and the initial invariant set $\Omega_{0}$ are selected, Algorithm 1 is fully automatic and no parameters need to be selected.

Now, applying Algorithm 1 with the controlled robust onestep operator given by Eq.(17), starting from an initial set $\Omega_{0}$, we get a sequence of convex sets that converges to the maximal controlled robust invariant set for the cancer system (4).

In Figure 2, the sequence of nested controlled robust invariant sets that converge to the maximal controlled robust invariant set is depicted in black line whereas the maximal controlled robust invariant set is depicted in blue line. This sequence of polytopes are computed starting from the set $\Omega_{0}=\left\{x \in X: x_{1} \geq a\right\}$ with $a$ big enough, for which the invariance condition $\Omega_{0} \subseteq Q_{W}\left(\Omega_{0}, X, U\right)$ holds. Consequently, the controlled robust one-step operator can be used to check controlled robust invariance.

Notice that $\Omega_{k+1}$ is defined as the set that contains all the states for which there exists a set of admissible chemotherapy drug such that these states will be mapped in $\Omega_{k}$ in one step regardless of state proportional uncertainties. In the same figure, the maximal controlled invariant set for the cancer system (4) is depicted in red line.

Figure 3 shows the maximal controlled robust invariant set in blue line and the maximal controlled invariant set in red line. Notice that the maximal controlled robust invariant set is calculated using Algorithm 1 starting from the set of state constraints $X$. The sequence of polytopes is depicted in black line.

By applying the change of variable defined above to the sets given in Figure 2, one gets the tumor-normal cells domains of attraction of the cancer system (2), that are depicted in Figure 4. In blue line, the domain of attraction of the uncertain cancer system (2) is showed. This contains all the tumor and normal states that can be driven to the set of healthy states considering that the dynamical cancer chemotherapy model is affected by state proportional uncertainties. In the same figure, the tumor-normal cells domain of attraction of the cancer system (2) without the state proportional uncertainties is given by the red line.

Remark from Figure 4 the drastic reduction of the domain of attraction caused by the state proportional uncertainties. Therefore, in order to achieve a successful chemotherapy treatment, the identification of the real values of the model parametersis crucial.

These domains of attraction represent, with arbitrary precision, the exact set of all the initial tumor and normal states for which there exists a specified set of administration therapy profile. This therapy leads to the eradication of the tumor cells without violating the constraint on the patient's health. Therefore, given the initial state of a patient, one could infer on the existence of a successful chemotherapy treatment. Furthermore, the amount of drugs that should be delivered 
and the therapy length can be calculated.

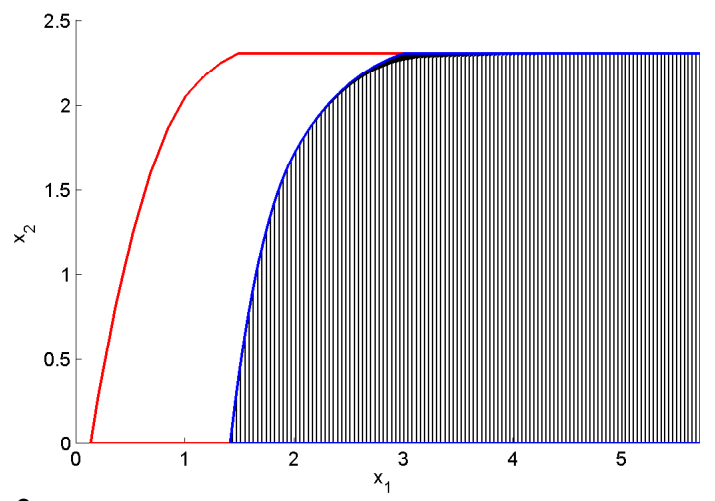

Fig. 2: Comparison between the maximal controlled invariant polyhedral set (red line) and the maximal controlled robust invariant polyhedral set (blue line) provided with polyhedral sequences considering state proportional uncertainty $w \in W=\left\{w \in \mathbb{R}^{2}:\|w\|_{2} \leqslant 0.06 ; 0 \leqslant w_{1} ; w_{2} \leqslant 0\right\}$ (black line), computed starting from an initial set $\Omega_{0}$. The admitted level of $x_{2}$ is equivalent to the admitted level of the normal cells, which is $10^{-1} \theta_{N}$ cells. The chemotherapy drugs are constrained to take value between $u_{\max }=1$ and $u_{\min }=0$.

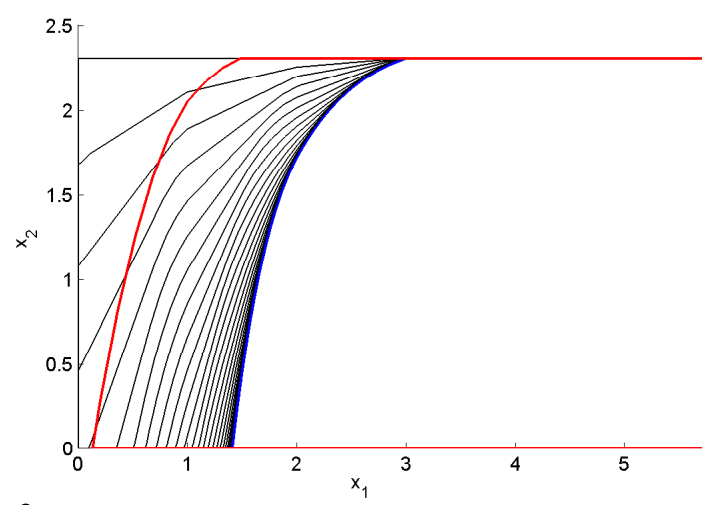

Fig. 3: Comparison between the maximal controlled invariant polyhedral set (red line) and the maximal controlled robust invariant polyhedral set (blue line) provided with polyhedral sequences considering state proportional uncertainty $w \in W=\left\{w \in \mathbb{R}^{2}:\|w\|_{2} \leqslant 0.06 ; 0 \leqslant w_{1} ; w_{2} \leqslant 0\right\}$ (black line), computed starting from the state constraints set $X$. The admitted level of $x_{2}$ is equivalent to the admitted level of the normal cells, which is $10^{-1} \theta_{N}$ cells. The chemotherapy drugs are constrained to take value between $u_{\max }=1$ and $u_{\min }=0$.

\section{CONCLUSION}

In this paper, we developed a method to determine the domain of attraction for cancer chemotherapy model. This domain contains all the initial tumor and normal states for which there exists a set of admissible drug administration therapy. This method could be used to characterize which patients can be successfully cured. This domain is obtained in the presence of state proportional uncertainties affecting the model and saturation constraints which limit the amount of the drug injection. In future work, alternative modelling frameworks could be employed.

\section{REFERENCES}

[1] E. Afenya. Acute leukemia and chemotherapy: a modeling viewpoint. Mathematical biosciences, 138(2):79-100, 1996.

[2] E. K. Afenya and D. E. Bentil. Some perspectives on modeling leukemia. Mathematical biosciences, 150(2):113-130, 1998.

[3] M. Alamir. Robust feedback design for combined therapy of cancer. Optimal Control Applications and Methods, 35(1):77-88, 2014.

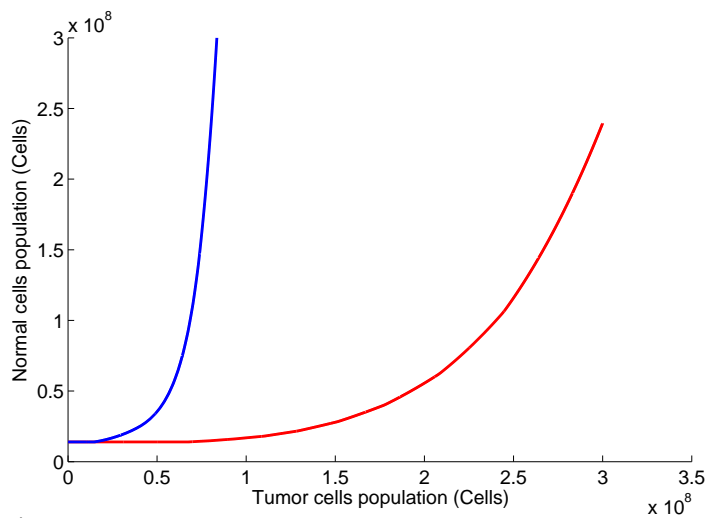

Fig. 4: Comparison between the domain of attraction of the deterministic cancer model (red line) and the domain of attraction of the cancer model (blue line) affected by state proportional uncertainty $w \in W=\left\{w \in \mathbb{R}^{2}:\|w\|_{2} \leqslant 0.06 ; 0 \leqslant w_{1} ; w_{2} \leqslant 0\right\}$. The admitted level of the normal cells is $10^{-1} \theta_{N}$ cells. The chemotherapy drugs are constrained to take value between $u_{\max }=1$ and $u_{\min }=0$.

[4] M. Alamir. On probabilistic certification of combined cancer therapies using strongly uncertain models. arXiv preprint arXiv:1502.06218, 2015.

[5] D. P. Bertsekas. Infinite-time reachability of state-space regions by using feedback control. IEEE Transactions on Automatic Control, 17:604-613, 1972.

[6] F. Blanchini and S. Miani. Set-Theoretic Methods in Control. Birkhäuser, 2008.

[7] S. Chareyron and M. Alamir. Mixed immunotherapy and chemotherapy of tumors: Feedback design and model updating schemes. Journal of theoretical biology, 258(3):444-454, 2009.

[8] L. G. de Pillis, W. Gu, K. R. Fister, T. Head, K. Maples, A. Murugan, T. Neal, and K. Yoshida. Chemotherapy for tumors: An analysis of the dynamics and a study of quadratic and linear optimal controls. Mathematical Biosciences, 209(1):292-315, 2007.

[9] L. G. de Pillis, W. Gu, and A. E. Radunskaya. Mixed immunotherapy and chemotherapy of tumors: modeling, applications and biological interpretations. Journal of theoretical biology, 238(4):841-862, 2006.

[10] M. Fiacchini. Convex difference inclusions for systems analysis and design. $\mathrm{PhD}$ thesis, Universidad de Sevilla, Spain, January 2010.

[11] M. Fiacchini, T. Alamo, and E. F. Camacho. On the computation of convex robust control invariant sets for nonlinear systems. Automatica, 46(8):1334-1338, 2010.

[12] M. Fiacchini, T. Alamo, and E. F. Camacho. Invariant sets computation for convex difference inclusions systems. Systems \& Control Letters, 61(8):819-826, 2012.

[13] E. G. Gilbert and K. Tan. Linear systems with state and control constraints: The theory and application of maximal output admissible sets. IEEE Transactions on Automatic Control, 36:1008-1020, 1991.

[14] P. Hahnfeldt, D. Panigrahy, J. Folkman, and L. Hlatky. Tumor development under angiogenic signaling a dynamical theory of tumor growth, treatment response, and postvascular dormancy. Cancer research, 59(19):4770-4775, 1999.

[15] I. Kolmanovsky and E. G. Gilbert. Theory and computation of disturbance invariant sets for discrete-time linear systems. Mathematical Problems in Engineering, 4:317-367, 1998.

[16] U. Ledzewicz, H. Schättler, and A. d'Onofrio. Optimal control for combination therapy in cancer. In $C D C$, pages 1537-1542, 2008.

[17] A. Matthias, S. Olaf, and B. Martin. Reachability analysis of nonlinear systems with uncertain parameters using conservative linearization. In Proceedings of the 47th IEEE Conference on Decision and Control, pages 4042-4048, 2008.

[18] A. S. Matveev and A. V. Savkin. Application of optimal control theory to analysis of cancer chemotherapy regimens. Systems \& control letters, 46(5):311-321, 2002.

[19] R. Riah, M. Fiacchini, and M. Alamir. Invariance-based analysis of cancer chemotherapy. In 2015 IEEE Conference on Control Applications (CCA), pages 1111-1116. IEEE, 2015.

[20] R. T. Rockafellar. Convex Analysis. Princeton University Press, USA, 1970.

[21] K. Shahab, M. John, O. Meeko, M. Ian M, and D. Guy A. Computing the viability kernel using maximal reachable sets. In Proceedings of the 15th ACM international conference on Hybrid Systems: Computation and Control, pages 55-64. ACM, 2012. 